\title{
Effect of Fluid Shear Stress on Endocytosis of Heparan Sulfate and Low-density Lipoproteins
}

\author{
Irmeli Barkefors, ${ }^{1,2}$ Cyrus K. Aidun, ${ }^{1}$ and E. M. Ulrika Egertsdotter ${ }^{3}$ \\ ${ }^{1}$ Department of Biomedical Engineering, George W. Woodruff School of Mechanical Engineering, \\ Georgia Institute of Technology, Atlanta, GA 30332, USA \\ ${ }^{2}$ Department of Medical Biochemistry and Microbiology, Uppsala University, 75123 Uppsala, Sweden \\ ${ }^{3}$ Biotechnology and Genetics program, College of Natural Resources, Virginia Polytechnic Institute and State University, Blacksburg, \\ VA 24061, USA
}

Correspondence should be addressed to E. M. Ulrika Egertsdotter, uegertsd@vt.edu

Received 30 April 2007; Revised 9 October 2007; Accepted 11 December 2007

Recommended by Ali A. Khraibi

Hemodynamic stress is a critical factor in the onset of atherosclerosis such that reduced rates of shear stress occurring at regions of high curvature are more prone to disease. The level of shear stress has direct influence on the thickness and integrity of the glycocalyx layer. Here we show that heparan sulfate, the main component of the glycocalyx layer, forms an intact layer only on cell surfaces subjected to shear, and not under static conditions. Furthermore, receptor-mediated endocytosis of heparan sulfate and low-density liporoteins is not detectable in cells exposed to shear stress. The internalized heparan sulfate and low-density lipoproteins are colocalized as shown by confocal imaging.

Copyright (c) 2007 Irmeli Barkefors et al. This is an open access article distributed under the Creative Commons Attribution License, which permits unrestricted use, distribution, and reproduction in any medium, provided the original work is properly cited.

\section{INTRODUCTION}

Atherosclerosis appears as a focal inflammatory disease at arterial tree bifurcations and junctions, as well as in regions with high curvature. Mechanical stresses due to blood flow are of major pathophysiological importance throughout the atherosclerotic disease process [1]. The relatively straight regions in the arteries, with high hemodynamic shear stress ( $>10$ dynes $/ \mathrm{cm}^{2}$ ), show higher resistance to disease development compared to bifurcation regions or sections with high curvature where flow separation and recirculation result in low-average shear stress [2] or low-oscillatory shear index [3].

The hemodynamic stress stimuli are transmitted through the endothelial cell surface layer, the glycocalyx layer, to reach the endothelial cell surface and generate an intracellular response $[4,5]$. There is evidence indicating that the glycocalyx has an important role as a transducer of mechanical forces to the intracellular cytoskeleton in the initiation of intracellular signaling [6]. In both micro- and macrovasculature, it have been demonstrated that constituents of the glycocalyx are involved in vascular homeostasis, such as maintaining the vascular permeability barrier, regulating the release of nitric ox- ide, and harboring a wide array of enzymes that might contribute to its vasculoprotective effect [6]. The glycocalyx layer is an organized mesh of membranous glycoproteins, proteoglycans, glycosaminoglycans (GAGs), and associated plasma proteins that can reach up to $3 \mu \mathrm{m}$ into the vessel lumen [7]. Different components of the glycocalyx layer play different roles during mechanotransduction [4]. Heparan sulfate, the most common GAG associated with 50 to $90 \%$ of the proteoglycans, has been shown to act like a mechanosensor for shear stress [8]. Shear stress has also been increasing the incorporation of hyaluronic acid into the glycocalyx [9].

It has been suggested that the glycocalyx layer physically shield the endothelial cell surfaces from attachment and uptake of damaging macromolecules in regions of low shear stress prone to vascular disease. The observed reduced thickness of the glycocalyx layer under low shear would then account for the higher rate of onset of disease in these regions [6]. Furthermore, by monitoring the shear stress-induced NO production, it was recently shown that the different glycocalyx components to different degrees diminish the effect of shear stress [4].

During noninflammatory conditions, macromolecules cross the endothelium by transcytosis that is a restricted 
direct transport through the endothelial cells, a process that requires endocytosis and exocytosis [10]. The transcytotic pathway appears to be affected by shear as indicated by shear dependence of albumin uptake in in vitro-cultured endothelial cells [11]. Macromolecules also pass across the endothelial monolayer through cell-cell junctions that can become "leaky" during inflammation when gaps are induced between endothelial cells at the level of adherens and tight junctional complexes [10]. The transport of albumin across an in vitro endothelial monolayer is acutely and reversibly sensitive to shear, possibly due to transient, shear-dependent widening of the intracellular junctions [12].

In the present study, we focus on the effects of shear stress on the thickness of the glycocalyx layer and uptake of LDL molecules into endothelial cells. The approach is to (1) characterize shear effects on the heparan sulfate component of the glycocalyx layer and (2) explore the hypothesis that the glycocalyx layer is shielding the endothelial cells by preventing active endocytosis of potentially hazardous bioactive molecules. In vitro experiments comparing the glycocalyx layer of live PAEC exposed to flow shear stress, to cells under static conditions, show significantly higher heparan sulfate staining on the surface of cells exposed to flow shear stress. Furthermore, the presence of heparan sulfate within the glycocalyx on the endothelial cell surface exposed to shear stress is associated with lower levels of lectin and LDL uptake in comparison to the cells exposed to no flow.

\section{MATERIAL AND METHOD}

\subsection{Cells medium and stains}

PAEC (Cell Application Inc., Calif, USA) at passages 1-5 were grown to confluency on fibronectin-coated cover slides in complete medium (EGM-MV, Cambrex, NJ, USA). Prior to introduction of the confluent cells into the flow chamber, the medium was changed to DMEM + Hepes (Cambrex) supplemented with $10 \%$ fetal bovine serum and $2 \mathrm{mM}$ L-glutamine. The flow experiments were conducted with this medium. Medium was also changed in control cells that were kept in stationary conditions.

The cells were stained for heparan sulfate using $10 \mu \mathrm{g} / \mathrm{mL}$ Texas Red- or FITC-conjugated lectins (Vector laboratories, Calif, USA) $10 \mu \mathrm{g} / \mathrm{mL}$ (Lycopersicon esculentum) in DPBS. $3 \mu \mathrm{M}$ Celltracker Orange (Cambrex) or $0.5 \mu \mathrm{g} / \mathrm{mL}$ Hoechst (Cambrex) was used as counter stain in some of the experiments. Staining was performed in stationary condition and samples were incubated for 60 minutes in $37^{\circ} \mathrm{C}$ or $4^{\circ} \mathrm{C}$. To prevent endocytosis, the cells were treated with $1 \mu \mathrm{M}$ phenyl arsine oxide (PAO) in serum-free medium (DMEM, Cambrex) for 45 minutes prior to staining and $1 \mu \mathrm{M}$ PAO was included in the staining solution.

\subsection{Shear stress experiments}

Cells were exposed to steady laminar shear stress for 24 or 48 hours in a perfusion chamber keeping a constant temperature of $37^{\circ} \mathrm{C}$ and a $5 \%$ concentration of $\mathrm{CO}_{2}$ in the medium. The height of the chamber was $2.5 \mathrm{~mm}$ and the flow rate
$10 \mathrm{~mL} / \mathrm{min}$, creating a wall shear stress in the middle of the channel equal to 11 dynes $/ \mathrm{cm}^{2}(1.1 \mathrm{~Pa})$. The flow profile was determined by computing the Poisuille profile in the channel and verifying the profile using microparticle image velocimeter, $\mu$ PIV (data not shown).

Cells were monitored during shear exposure using an inverted light microscope and images were taken using simple PCI imaging system. After removal from the chamber, the samples were washed in DPBS and stained. Images were collected using a Zeiss 510 confocal microscope and processed using Zeiss 510 image browser. All experiments were repeated three times.

\subsection{Heparinase treatment}

Following shear stress exposure, cells were treated with Heparinase III (Sigma-Aldrich, Miss, USA) by incubation with enzyme $(1 \mathrm{U} / \mathrm{mL})$ in $20 \mathrm{mM}$ Tris- $\mathrm{HCl}$ buffer $(\mathrm{pH} 7.5,4 \mathrm{mM}$ $\mathrm{CaCl}_{2}, 0.1 \mathrm{mg} / \mathrm{mL} \mathrm{BSA}$ ) for 45 minutes. Control cells were kept stationary and treated identically. The cells were then stained for heparan sulfate using Texas Red-labeled lectins as described above. A control sample was incubated with serum-free medium only and stained with lectin identically.

\subsection{Fixation and antibody staining}

Fixation of the cells was performed by $4 \%$ paraformaldehyde applied to the monolayer immediately after removal from the flow chamber. Cells were fixed for 15 minutes and washed 3 times for 10 minutes in DPBS containing $2 \mathrm{mM}$ glycine and stained for heparan sulphate using either lectins (LEA) or antiheparan/heparan sulfate human monoclonal antibodies (MAB2040, Chemicon, Millipore, Mass, USA). CellTracker Orange (Cambrex) was used to stain the cytoplasm.

\subsection{LDL uptake and immobilization}

After shear stress exposure for 24 or 48 hours, confluent PAEC were incubates with fluorescence-labeled low-density lipoprotein (DiI-LDL, L-3482, $10 \mu \mathrm{g} / \mathrm{mL}$, Invitrogen, Md, USA) and FITC-LEA (Vector laboratories, $10 \mu \mathrm{g} / \mathrm{mL}$ ) in DPBS for 60 minutes, $37^{\circ} \mathrm{C} 5 \% \mathrm{CO}_{2}$. For all the experiments, a control was kept in static flow conditions. The distribution of intracellular stain and stain immobilized on the surface was examined with confocal microscopy.

\section{RESULTS}

The effect of shear stress on the glycocalyx structure was examined by exposing PAEC to shear stress at $11 \mathrm{dynes} / \mathrm{cm}^{2}$ for 24 or 48 hours, in comparison to control cells subjected to no flow in static culture conditions. The glycocalyx layer was visualized by staining of live cells with Texas Red-conjugated lectin LEA specific for heparan sulfate, the main component of the glycocalyx layer. In cells subjected to shear stress (see Figures 1(a) and 1(b)), it is observed that heparan sulfate accumulates on the cell surface in addition to some intracellular staining. The layer of heparan sulfate stain on the cell surface is more promi- 


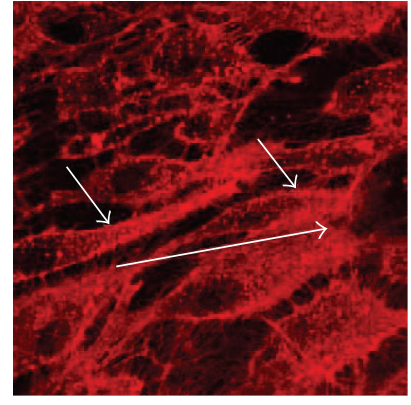

(a)

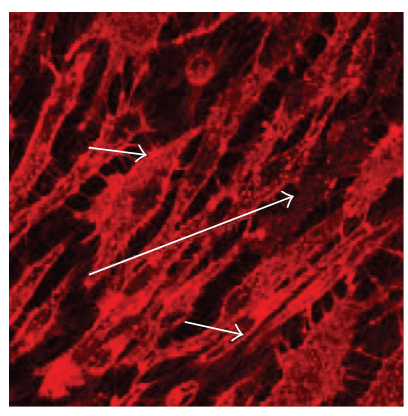

(b)

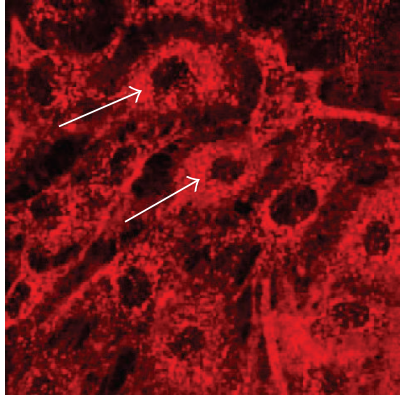

(c)

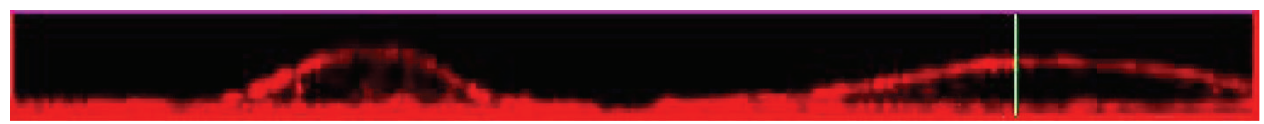

(d)

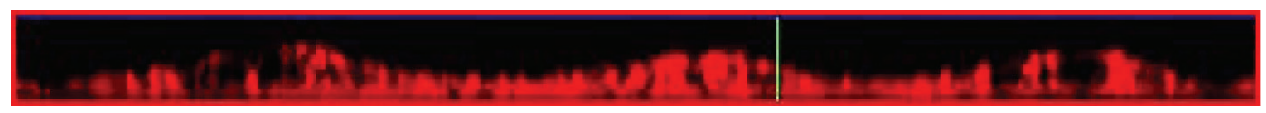

(e)

FIGURE 1: Effects from flow on the thickness and integrity of the glycocalyx layer of PAEC exposed to flow shear stress at 11 dynes/ $\mathrm{cm}^{2}$ and under static conditions for control. The glycocalyx layer is visualized under the confocal microscope through staining of heparan sulfate with Texas Red-conjugated lectin after different times in culture. (a) Cells exposed to flow shear stress for 24 hours, (b) cells exposed to flow shear stress for 48 hours, and (c) cells grown under static conditions for 24 hours. The images are cross-sections of the cell monolayer $z=0.5-2 \mu \mathrm{m}$ with $z=0$ at the glass surface. Large arrows indicate approximate flow directions. Small arrows indicate the distribution of the stain, predominantly located in the interior of the cells not exposed to shear stress compared to the shear-exposed cells where it is most abundant at the surface. Figures 1(d) and 1(e) are the cross-sections from the side of the stack of images created with confocal microscopy with (d) cells exposed to shear stress for 24 hours and (e) cells grown under static conditions for 24 hours.

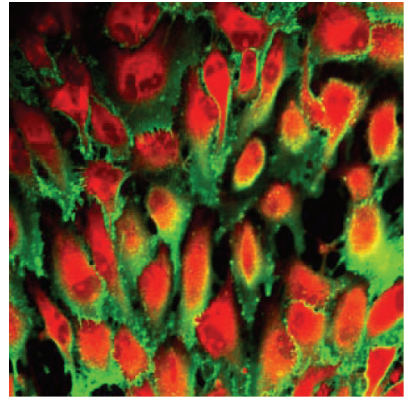

(a)

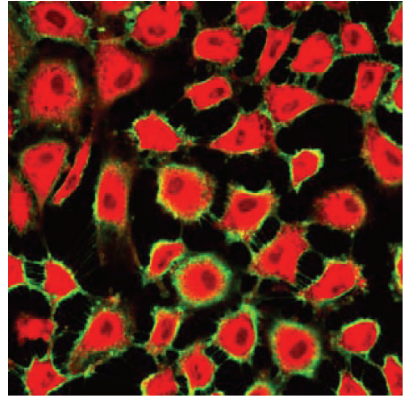

(b)

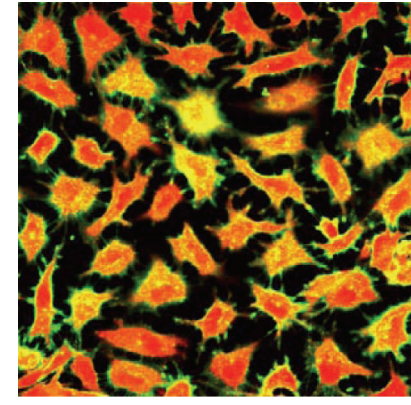

(c)

FIgURE 2: Role of endocytosis on the internalization of FITC-conjugated lectins. PAEC exposed to flow shear stress at $11 \mathrm{dynes} / \mathrm{cm}^{2}$ and under static conditions under different temperatures that are permissive for endocytosis $\left(37^{\circ} \mathrm{C}\right.$; control $)$ and nonpermissive $\left(+4^{\circ} \mathrm{C}\right)$. The glycocalyx layer is visualized under the confocal microscope through staining of heparan sulfate with FITC-conjugated lectin (green) and counter stained with CellTracker Orange (red) after different times in culture. (a) Cells exposed to flow shear stress for 24 hours at $+4^{\circ} \mathrm{C}$, (b) cells grown under static condition for 24 hours at $+4^{\circ} \mathrm{C}$, and (c) cells grown under static conditions for 24 hour at $37^{\circ} \mathrm{C}$. Internalized FITC stain appears yellow.

nent after 48 hours of shear stress (see Figure 1(b)). Cells grown under static condition show continuous staining of heparan sulfate throughout the cell except for the nucleus (see Figure 1(c)). When examining the cross-section of the confocal image stacks, small amounts of heparan sulfate stain are observed within the cells subjected to flow, and a clear layer of heparan sulfate stain on the cell surface (see Figure $1(\mathrm{~d})$ ). In cells grown under static condition, it is found that the heparan sulfate stain is dispersed within the cells (see Figure 1(e)).
The mechanism for translocation of heparan sulfate stain to the inside of the cells is investigated by performing the heparan sulfate experiments with treatments (low temperature or endocytosis inhibitor) that reduce or block active uptake by endocytosis. In these experiments, the cytoplasm is stained with CellTracker Orange to reveal the exact outline of the cells. To avoid overlap in fluorescence, FITC-conjugated lectin LEA is used to stain for heparan sulfate. Cells grown for 24 hours under shear stress at $+4^{\circ} \mathrm{C}$ or under static conditions at $37^{\circ} \mathrm{C}$ and $+4^{\circ} \mathrm{C}$ are stained for heparan sulfate and 


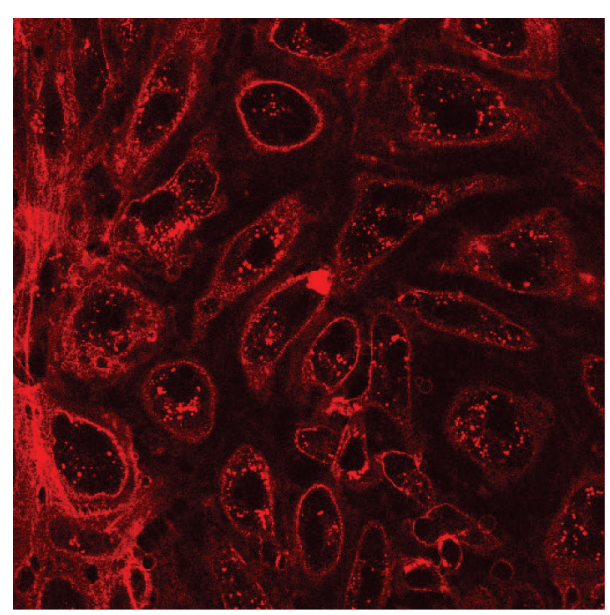

(a)

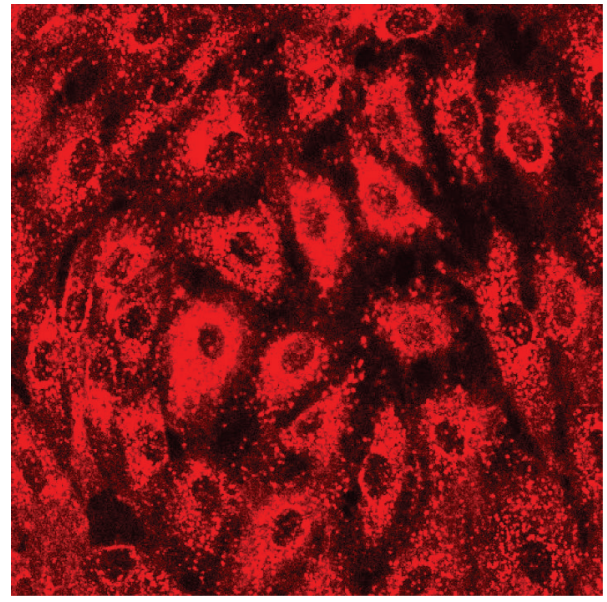

(b)

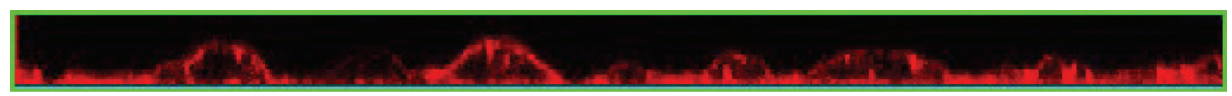

(c)

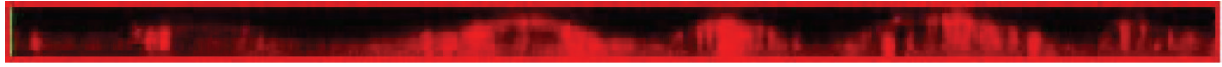

(d)

FIGURE 3: Effects of PAO on internalization of FITC-conjugated lectins. The GL is visualized under the confocal microscope through staining of heparan sulfate with Texas Red-conjugated lectin after 24 hours in culture. (a) Cells grown in static condition, 45 minutes prior to staining medium were changed to EMDM with $10 \mu \mathrm{M}$ Phenyl Arsine Oxide to prevent endocytosis, (b) cells grown under static condition. Images are cross-sections of the cell monolayer $z=0.5-2 \mu \mathrm{m}$ with $z=0$ at the glass surface, (c) cross-sections from the side of the stack of images of (a) created with confocal microscopy, and (d) cross-sections from the side of the stack of images of (b) created with confocal microscopy.

counterstained with CellTracker Orange. The results of these experiments show that cells are stained yellow for heparan sulfate (combination of FITC and CellTracker Orange) in the cell interior and green (FITC) on the outside (see Figure 2). Cells grown under shear for 24 hours at $4^{\circ} \mathrm{C}$ show significant presence of heparan sulfate on the outside of the cells (see Figure 2(a)). There are less staining for heparan sulfate on the outside of the cells grown under static condition at $4^{\circ} \mathrm{C}$ (see Figure $2(\mathrm{~b})$ ) or $37^{\circ} \mathrm{C}$ (see Figure 2(c)). Staining of the cells at $+4^{\circ} \mathrm{C}$ prevented the uptake of heparan sulfate almost completely in the cells subjected to shear stress (see Figure 2(a)) as well as in the control cells grown in static condition at $+4^{\circ} \mathrm{C}$ (see Figure $2(\mathrm{~b})$ ).

Additional experiments to verify the uptake of heparan sulfate by endocytosis is done by adding the endocytosis inhibitor PAO prior to staining with Texas Red-conjugated lectins. This treatment considerably reduces the internalization of stain in treated cells grown under static condition (see Figure 3(a)) compared to untreated control cells (see Figure $3(\mathrm{~b})$ ). The reduction in uptake by addition of PAO is confirmed by examination of the cross-section of the confocal stacks showing limited heparan sulfate inside the cells after PAO treatment (see Figure 3(c)), compared to uniform distribution throughout the cell interior in the control cells (see Figure 3(d)).

The heparan sulfate detected internally appears to be taken up through endocytosis as discussed above. To examine the effect from the presence of heparan sulfate in the glycocalyx layer on endocytosis, heparan sulfate is removed enzymatically from the cell surface before staining (see Figure 4). The detectable heparan sulfate on the outside of the heparinase-treated cells is significantly reduced in both cells subjected to flow for 24 hours (see Figure 4(a)) and in the static control cells (see Figure 4(b)), indicating successful removal of heparan sulfate from the cell surface. Staining, however, appeared on the inside of the control cells (see Figure 4(b)) similarly to nontreated cells with intact heparan sulfate layers (cf. Figure 1(c)). Addition of heparan sulfate to the control cells before staining also did not affect the uptake of stain (data not shown).

The long-term objective of this study is to gain insight in the effect of the glycocalyx layer on the uptake of LDL during the earlier stages of atherosclerosis in areas of low shear. To this end, the role of the glycocalyx layer on uptake of LDL by endothelial cells is examined in cells subjected to shear stress as well as control cells. Cells grown under shear stress for 24 hours, 48 hours, and under static condition are simultaneously incubated with fluorescence-tagged LDL (DiILDL) and heparan sulfate stain (FITC-LEA). The cells exposed to shear stress for 24 hours (Figure 5(a)) show significantly less macromolecule uptake compared to the control (see Figure 5(c)). The cells exposed to shear for 48 hours show almost no uptake of LDL (see Figure 5(b)). Crosssections of the confocal image stacks reveal the location of the LDL to the surface membrane after shear stress (see Figures 5(d) and 5(e) for 24 hours and 48 hours, resp.) and also to the 


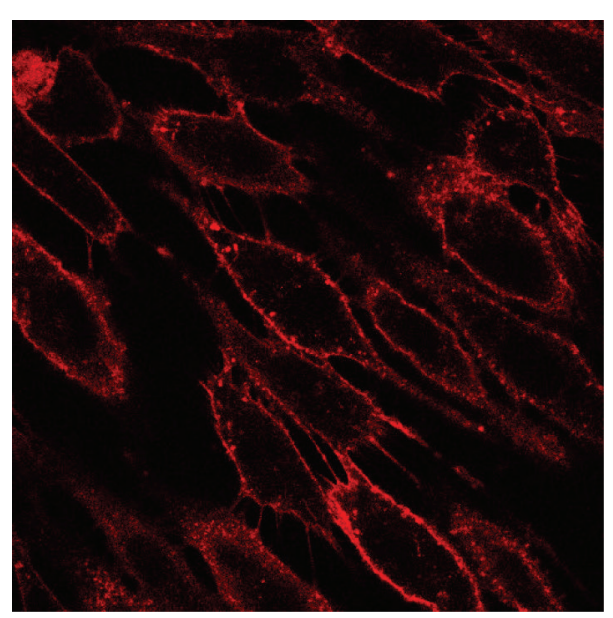

(a)

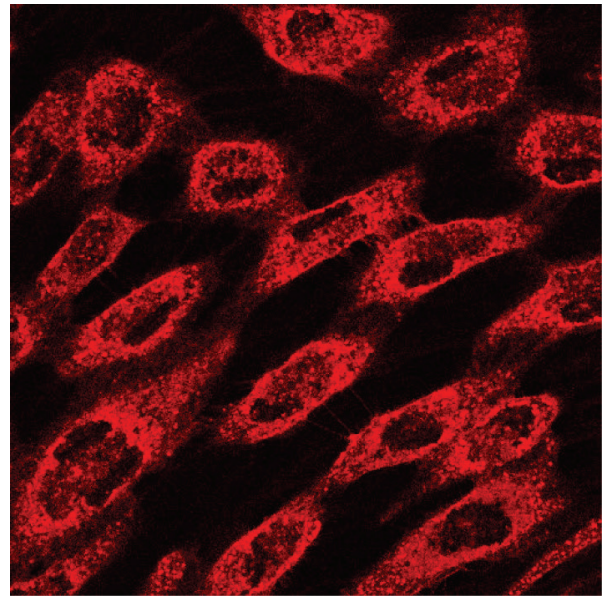

(b)

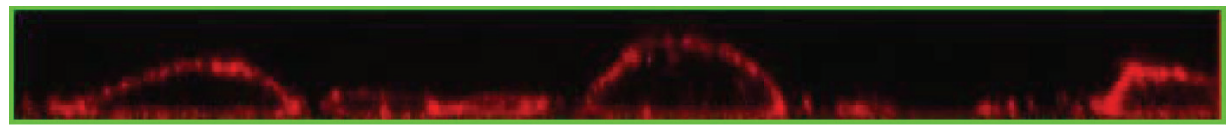

(c)

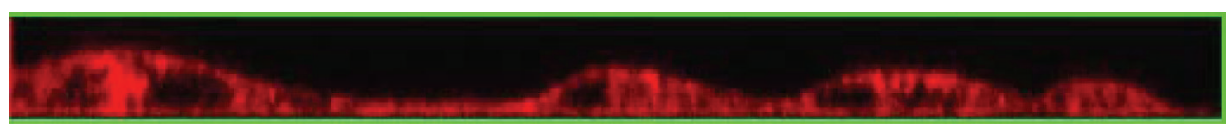

(d)

FIGURE 4: Effects of enzymatic removal of heparan sulfate before heparan sulfate staining of PAEC grown under different flow conditions for 24 hours. The glycocalyx layer is visualized under the confocal microscope through staining of heparan sulfate with Texas Red-conjugated lectin. (a) Cells grown for 24 hours exposed to laminar shear stress of 11 dynes $/ \mathrm{cm}^{2}$ and then treated with HepIII for 45 minutes before staining, (b) control cells grown under static conditions for 24 hours before treatment with HepIII staining. Images are cross-sections of the cell monolayer $z=0.5-2 \mu \mathrm{m}$ with $z=0$ at the glass surface, (c) cross-sections from the side of the stack of images of (a) created with confocal microscopy, and (d) cross-sections from the side of the stack of images of (b) created with confocal microscopy.

inside of the cells under static condition (see Figure 5(f)). Examination with multichannel confocal microscope revealed that a large proportion of the internalized LDL (red) were colocalized with the heparan sulfate stain (green) appearing yellow as seen in Figure 5(c).

Treatment of the cells with PAO, in addition to the LDL and heparan sulfate stain, reduced the internalization of both LDL and heparan sulfate stain (see Figure 6(a)) compared to the control without PAO (see Figure 6(b)) as confirmed by the cross-section images presented in Figures 6(c) and 6(d), respectively.

\section{DISCUSSION}

Considerable effort is underway to better understand the underlying pathophysiology of atherosclerosis and the fundamental biology and biomechanics of atherosclerotic lesion. In order to aid in clinical prevention, it is important to understand the mechanism through which lesion formation preferentially occurs in localized regions with low shear stress. It has been suggested that the surface layer of the endothelial cells, the glycocalyx layer, is playing a role in preventing the onset of vascular disease due to its shielding properties preventing inflammatory substances to reach the surface of the endothelial cell. Experimental data have con- firmed that the properties of the glycocalyx layer change under different shear stress regimes [7].

In the present study, we have utilized confocal imaging to investigate the effect of flow stress on the thickness, molecular composition, and function of the glycocalyx layer of in vitro endothelial cells. Accurate visualization of the glycocalyx layer of endothelial cell is challenging as common fixation procedures collapse part of the glycocalyx structure. The glycocalyx layer of endothelial cells has, however, been mostly examined by staining after fixation of in vitro or in vivo endothelial cells $[7,11,13]$. To obtain a more accurate view of the complete glycocalyx layer, we have used live cells to analyze the structure and thickness of the glycocalyx layer by staining for heparan sulfate.

It has previously been shown by cell culture studies that the thickness of the glycocalyx layer increases by $74 \%$ when the laminar shear stress is increased from $1.0 \mathrm{~Pa}$ to $3.0 \mathrm{~Pa}$ [11]. Furthermore, shear stress at $40 \mathrm{dynes} / \mathrm{cm}^{2}$ for 24 hours significantly increased GAG synthesis and the presence of GAGs on the cell surface and in the solubilized matrix, suggesting that the higher levels of heparan sulfate under shear stress is partly accounted for by unbound molecules associated with the glycocalyx layer [14]. The level of shear stress has also been shown to have a positive relation to glycocalyx layer thickness in vivo in mice aorta, such that endothelial 


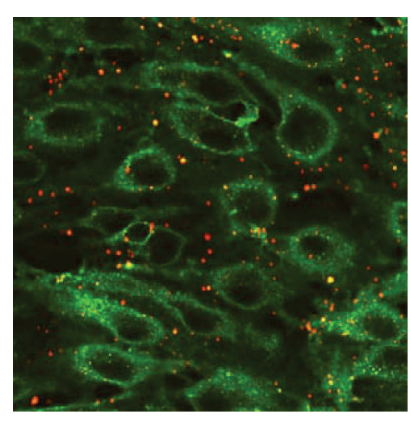

(a)

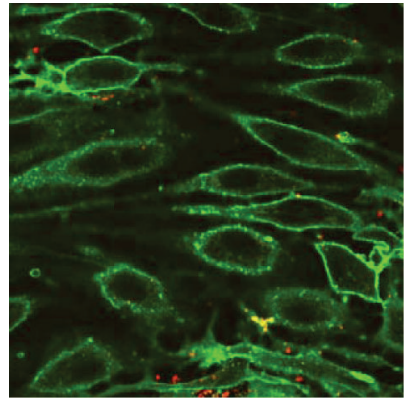

(b)

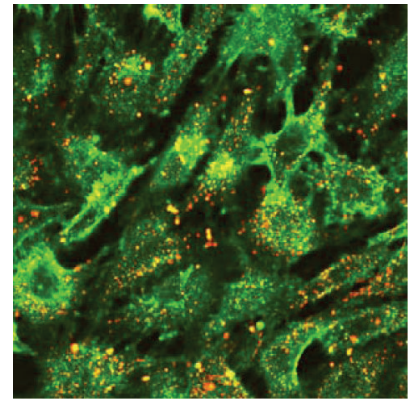

(c)

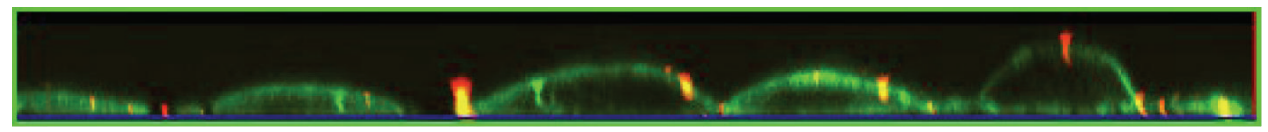

(d)

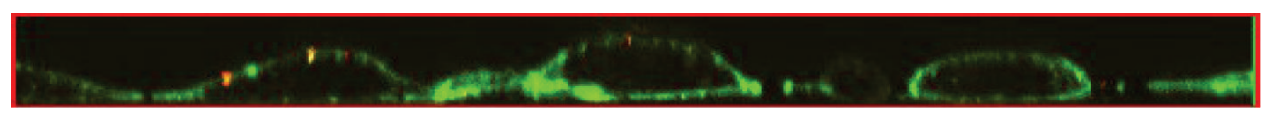

(e)

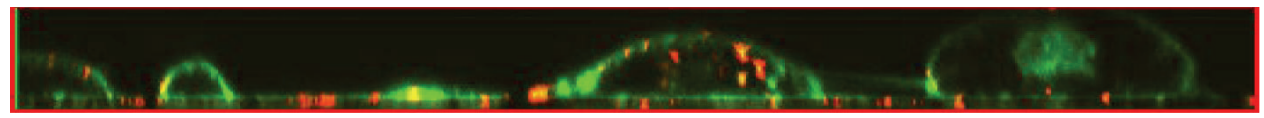

(f)

FIGURE 5: Flow effects on the glycocalyx layer and uptake of LDL. PAEC incubated with LDL after exposure to different conditions of flow. The glycocalyx layer is visualized under the confocal microscope through staining of heparan sulfate with FITC-conjugated lectin (green) and LDL added as DiI complex (red) after different times in culture. (a) Cells exposed to flow shear stress for 24 hours, (b) 48 hours, and (c) cells grown under static conditions for 24 hours. Internalized LDL appears yellow when colocalized with heparan sulfate stain. (d) Crosssection from the image stacks created with confocal microscopy of the stack (a), (e) cross-section from the side of the stack (b), and (f) cross-sections from the side of the stack (c).

cells exposed to higher levels of average laminar shear stress in mice aorta show thicker glycocalyx layer [13]. In the present study, the glycocalyx layer of endothelial cells was observed after various time periods at one shear stress level, compared to static conditions. Here, 11 dynes $/ \mathrm{cm}^{2}$ shear stress for 24 hours was shown to promote the formation of a continuous layer of heparan sulfate on the surface of endothelial cells. The heparan sulfate layer became more prominent after 48 hours of exposure to shear stress. By contrast, in cells grown without shear under stationary conditions, heparan sulfate was only detected at intracellular locations and not on the surface of the endothelial cells. In agreement with previous studies, our data suggests that there is a positive relation between the thickness of the glycocalyx layer and the level and amount of shear sensed by the endothelial cells.

Observations during the earlier stages of atherosclerosis have shown that there is an accumulation of macromolecules, especially low-density lipoproteins (LDL), in areas of low hemodynamic shear associated with atherosclerotic plaque development. The passage of LDL through the endothelial monolayer has been suggested to occur through leaky junctions and by transcytosis, referred to, respectively, as "transport" and "uptake" in this study. It was recently shown that under pressurized "convective" conditions, $90 \%$ of the LDL transport across the endothelial monolayer is through "leaky" junctions associated with dying or dividing cells [15]. The effect of shear stress on transendothelial transport of albumin shows that the transport is acutely and reversibly sensitive to shear stress. The permeability increases almost by a factor of two in response to shear stress applied at 10 dynes $/ \mathrm{cm}^{2}$ compared to 1 dyne $/ \mathrm{cm}^{2}[12]$.

The uptake of macromolecules by receptor-mediated transcytosis has been studied under shear stress [11, 16, 17]. The uptake of albumin into bovine aortic endothelial cells (BAEC) under shear stress was lower at $3.0 \mathrm{~Pa}$ relative to $1.0 \mathrm{~Pa}$ and relative to static conditions [11]. Internalization of radioactively labeled LDL was studied in BAEC exposed to 24 hours pretreatment of shear stress at 30 dynes $/ \mathrm{cm}^{2}$ or less than 1 dyne $/ \mathrm{cm}^{2}$. While continuously under shear, LDL was then added to the cells and incubated under the same respective pretreatment shear stresses for 2 or 24 hours. The uptake of LDL was highest after 24 hours of incubation with LDL in the BAEC that had been exposed to the pretreatment shear stress of 30 dynes $/ \mathrm{cm}^{2}$ [16]. The stimulatory effect of shear stress on LDL uptake was recently confirmed in another set of experiments where confluent BAEC showed a higher LDL uptake when incubated with LDL under shear stress of 1.0 Pa compared to LDL uptake under static conditions [17]. In the present study, PAEC pretreated with shear stress at 11 dynes $/ \mathrm{cm}^{2}$ for 24 hours and subsequently incubated with LDL for 1 hour under static condition showed 


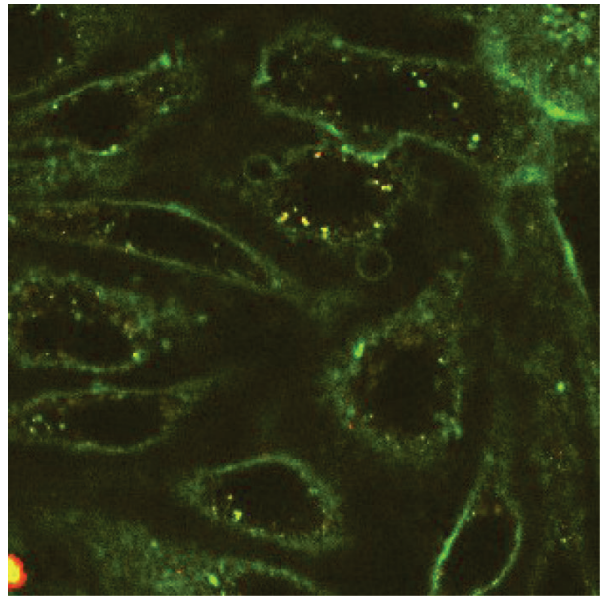

(a)

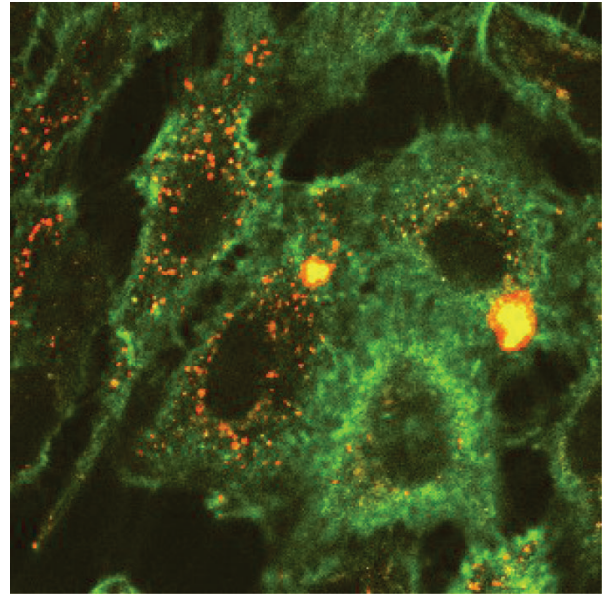

(b)

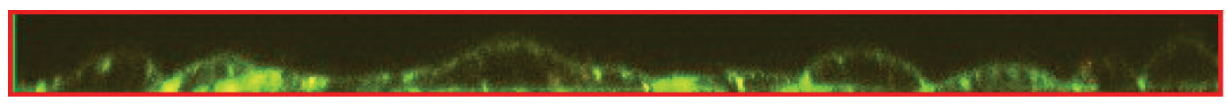

(c)

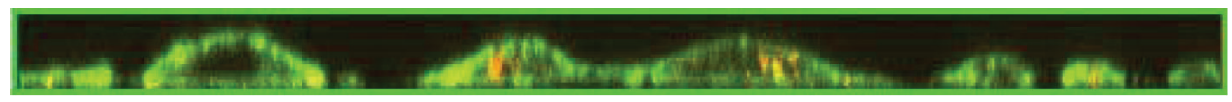

(d)

FIGURE 6: Effect of PAO on uptake of LDL by PAEC grown under static conditions. The glycocalyx layer is visualized under the confocal microscope through staining of heparan sulfate with FITC-conjugated lectin (green) and LDL added as DiI complex (red) after different times in culture. (a) Cells grown under static conditions for 24 hours and incubated with PAO together with FITC-conjugated lectins and (b) cells grown under static conditions for 24 hours. Internalized LDL appear yellow when colocalized with heparan sulfate stain. (c) Crosssections of image stacks created with confocal microscopy of (a) and (d) cross-sections from the side of the stack of images of (b).

less LDL uptake than control cells pretreated for 24 hours and incubated for 1 hour under static condition. It has been previously shown that albumin uptake in BAEC is not significantly affected by the uptake condition; such that the uptake under static conditions and under continuous shear stress equally reflect the pretreatment shear stress conditions that the cells were exposed to prior to the uptake experiment [11]. For albumin, the uptake is, however, lower at shear stress of 30 dynes $/ \mathrm{cm}^{2}$, and higher at 10 dynes $/ \mathrm{cm}^{2}$, relative static conditions [16] as opposed to the increasing uptake observed for LDL under shear stress [17]. The data presented here show lower uptake of LDL in cells that have been exposed to shear stress of 11 dynes $/ \mathrm{cm}^{2}$, compared to LDL uptake in cultures exposed to static conditions. However, the uptake experiments in this study were carried out under static conditions as opposed to the previous studies on LDL uptake $[16,17]$ which measured the uptake under continuous shear stress.

The present study shows that cells exposed to shear stress at 11 dynes $/ \mathrm{cm}^{2}$ for 24 hours develop a border lining of heparan sulfate (see Figure 1(a)) and that the continuous layer of heparan sulfate on the endothelial cell surface is associated with limited uptake of LDL (see Figure 5(a)) in comparison to cells grown under static conditions lacking a heparan sulfate layer (see Figures 1(c) and 5(c), resp.). The protective role of the glycocalyx layer and heparan sulfate for the onset of vascular disease by LDL uptake is complicated by the finding that cellular uptake of LDL by endothelial cells initiates through binding to heparan sulfate followed by receptor-mediated endocytosis [18]. By contrast, the receptor for LDL have been found to be inhibited by sulphonated glucosaminoglycans, like heparan sulfate $[19,20]$. Duan et al. [21] have found that an increase in cell surface HSPG reduces atherogenic events including lipoprotein transport. In PAEC, grown under static conditions, we detected intracellular colocalization of the stains for LDL and heparan sulfate suggesting that LDL and heparan sulfate are endocytosed as a complex (see Figure 6).

The nonoxidized LDL used in these experiments is predicted to bind the lectin-like LDL receptor and not the scavenger receptor that is responsible for uptake of oxidized LDL. We cannot exclude that the added LDL are oxidized on the cell surface where oxidative agents are released [22] although it has been suggested that oxidation of LDL to a large extent occurs below the endothelial cell layer [23].

The function of different types of LDL receptors implicated in the development of atherosclerosis is still also unclear. Lectin-like, oxidized low-density lipoprotein receptor1 (LOX-1) has been observed at elevated levels in atherosclerotic areas [24], whereas another member of the LDL receptor family (low-density receptor-related protein) has been suggested to protect the vascular wall against atherosclerosis [25].

Experimental studies on the actual uptake of macromolecules into in vitro-cultured endothelial cells subjected 
to shear stress are conflicting possibly due to different experimental conditions that may trigger different receptor mechanisms. Here we show experimentally that LDL uptake under static conditions into the endothelial cells by vesicular transport is occurring less frequently in cells that have been previously exposed to shear stress. Future more detailed studies on the receptor mechanisms involved in shear-dependent LDL uptake will be necessary to fully understand the function and effect of LDL uptake in the development of atherosclerotic lesion in areas of low shear stress, as well as confirmation by in vivo experiments of existing experimental data and mechanisms suggested by model simulations.

\section{REFERENCES}

[1] D. P. Giddens, C. K. Zarins, and S. Glagov, "The role of fluid mechanics in the localization and detection of atherosclerosis," Journal of Biomechanical Engineering, vol. 115, no. 4 B, pp. 588-594, 1993.

[2] D. N. Ku, D. P. Giddens, D. J. Phillips, and D. E. Strandness Jr., "Hemodynamics of the normal human carotid bifurcation: in vitro and in vivo studies," Ultrasound in Medicine and Biology, vol. 11, no. 1, pp. 13-26, 1985.

[3] D. N. Ku, D. P. Giddens, C. K. Zarins, and S. Glagov, "Pulsatile flow and atherosclerosis in the human carotid bifurcation. Positive correlation between plaque location and low and oscillating shear stress," Arteriosclerosis, vol. 5, no. 3, pp. 293302, 1985.

[4] M. Y. Pahakis, J. R. Kosky, R. O. Dull, and J. M. Tarbell, “The role of endothelial glycocalyx components in mechanotransduction of fluid shear stress," Biochemical and Biophysical Research Communications, vol. 355, no. 1, pp. 228-233, 2007.

[5] S. Weinbaum, X. Zhang, Y. Han, H. Vink, and S. C. Cowin, "Mechanotransduction and flow across the endothelial glycocalyx," Proceedings of the National Academy of Sciences of the United States of America, vol. 100, no. 13, pp. 7988-7995, 2003.

[6] M. M. Thi, J. M. Tarbell, S. Weinbaum, and D. C. Spray, "The role of the glycocalyx in reorganization of the actin cytoskeleton under fluid shear stress: a "bumper-car" model," Proceedings of the National Academy of Sciences of the United States of America, vol. 101, no. 47, pp. 16483-16488, 2004.

[7] M. Gouverneur, B. Van Den Berg, M. Nieuwdorp, E. Stroes, and H. Vink, "Vasculoprotective properties of the endothelial glycocalyx: effects of fluid shear stress," Journal of Internal Medicine, vol. 259, no. 4, pp. 393-400, 2006.

[8] J. A. Florian, J. R. Kosky, K. Ainslie, Z. Pang, R. O. Dull, and J. M. Tarbell, "Heparan sulfate proteoglycan is a mechanosensor on endothelial cells," Circulation Research, vol. 93, no. 10, pp. e136-142, 2003.

[9] M. Gouverneur, J. A. E. Spaan, H. Pannekoek, R. D. Fontijn, and H. Vink, "Fluid shear stress stimulates incorporation of hyaluronan into endothelial cell glycocalyx," American Journal of Physiology-Heart and Circulatory Physiology, vol. 290, no. 1, pp. H458-H462, 2006.

[10] G. Hu, A. T. Place, and R. D. Minshall, "Regulation of endothelial permeability by Src kinase signaling: vascular leakage versus transcellular transport of drugs and macromolecules," Chemico-Biological Interactions. In press.

[11] A. Ueda, M. Shimomura, M. Ikeda, R. Yamaguchi, and K. Tanishita, "Effect of glycocalyx on shear-dependent albumin uptake in endothelial cells," American Journal of PhysiologyHeart and Circulatory Physiology, vol. 287, pp. H2287-H2294, 2004.
[12] H. Jo, R. O. Dull, T. M. Hollis, and J. M. Tarbell, "Endothelial albumin permeability is shear dependent, time dependent, and reversible," American Journal of Physiology-Heart and Circulatory Physiology, vol. 260, p. 5, 1991.

[13] B. M. Van Den Berg, J. A. E. Spaan, T. M. Rolf, and H. Vink, "Atherogenic region and diet diminish glycocalyx dimension and increase intima-to-media ratios at murine carotid artery bifurcation," American Journal of Physiology-Heart and Circulatory Physiology, vol. 290, no. 2, pp. H915-H920, 2006.

[14] T. Arisaka, M. Mitsumata, M. Kawasumi, T. Tohjima, S. Hirose, and Y. Yoshida, "Effects of shear stress on glycosaminoglycan synthesis in vascular endothelial cells," Annals of the New York Academy of Sciences, vol. 748, no. 1, pp. 543-554, 1995.

[15] L. M. Cancel, A. Fitting, and J. M. Tarbell, "In vitro study of LDL transport under pressurized (convective) conditions," American Journal of Physiology - Heart and Circulatory Physiology, vol. 293, no. 1, pp. H126-H132, 2007.

[16] E. A. Sprague, B. L. Steinbach, R. M. Nerem, and C. J. Schwartz, "Influence of a laminar steady-state fluid-imposed wall shear stress on the binding, internalization, and degradation of low-density lipoproteins by cultured arterial endothelium," Circulation, vol. 76, no. 3, pp. 648-656, 1987.

[17] M. Traoré, R. J. Sun, S. Fawzi-Grancher, et al., "Kinetics of the endocytotic pathway of low density lipoprotein (LDL) in human endothelial cells line under shear stress: an in vitro confocal microscopy study," Clinical Hemorheology and Microcirculation, vol. 33, no. 3, pp. 243-251, 2005.

[18] M. Mulder, P. Lombardi, H. Jansen, T. J. C. Van Berkel, R. R. Frants, and L. M. Havekes, "Low density lipoprotein receptor internalizes low density and very low density lipoproteins that are bound to heparan sulfate proteoglycans via lipoprotein lipase," Journal of Biological Chemistry, vol. 268, no. 13, pp. 9369-9375, 1993.

[19] M. S. Brown and J. L. Goldstein, "Receptor-mediated endocytosis: insights from the lipoprotein receptor system," Proceedings of the National Academy of Sciences of the United States of America, vol. 76, no. 7, pp. 3330-3337, 1979.

[20] E. Vasile, M. Simionescu, and N. Simionescu, "Visulaization of the binding, endocytosis and transcytosis of low-density lipoprotein in the arterial endothelium in situ," Journal of Cell Biology, vol. 96, pp. 1677-1689, 1983.

[21] W. Duan, L. Paka, and S. Pillarisetti, "Distinct effects of glucose and glucosamine on vascular endothelial and smooth muscle cells: evidence for a protective role for glucosamine in atherosclerosis," Cardiovascular Diabetology, vol. 4, p. 16, 2005.

[22] D. Steinberg, "Low denisty lipoprotein oxidation and its pathological significance," The Journal of Biological Chemistry, vol. 272, no. 34, p. 4, 1997.

[23] A. C. Carr, M. R. McCall, and B. Frei, "Oxidation of LDL by myeloperoxidase and reactive oxygene species: reaction pathways and antioxidante protection," Arteriosclerosis, Thrombosis, and Vascular Biology, vol. 20, pp. 1716-1723, 2000.

[24] J. L. Mehta, J. Chen, P. L. Hermonat, F. Romeo, and G. Novelli, "Lectin-like, oxidized low-density lipoprotein receptor-1 (LOX-1): a critical player in the development of atherosclerosis and related disorders," Cardiovascular Research, vol. 69, no. 1, pp. 36-45, 2006.

[25] P. Boucher and M. Gotthardt, "LRP and PDGF signaling: a pathway to atherosclerosis," Trends in Cardiovascular Medicine, vol. 14, no. 2, pp. 55-60, 2004. 

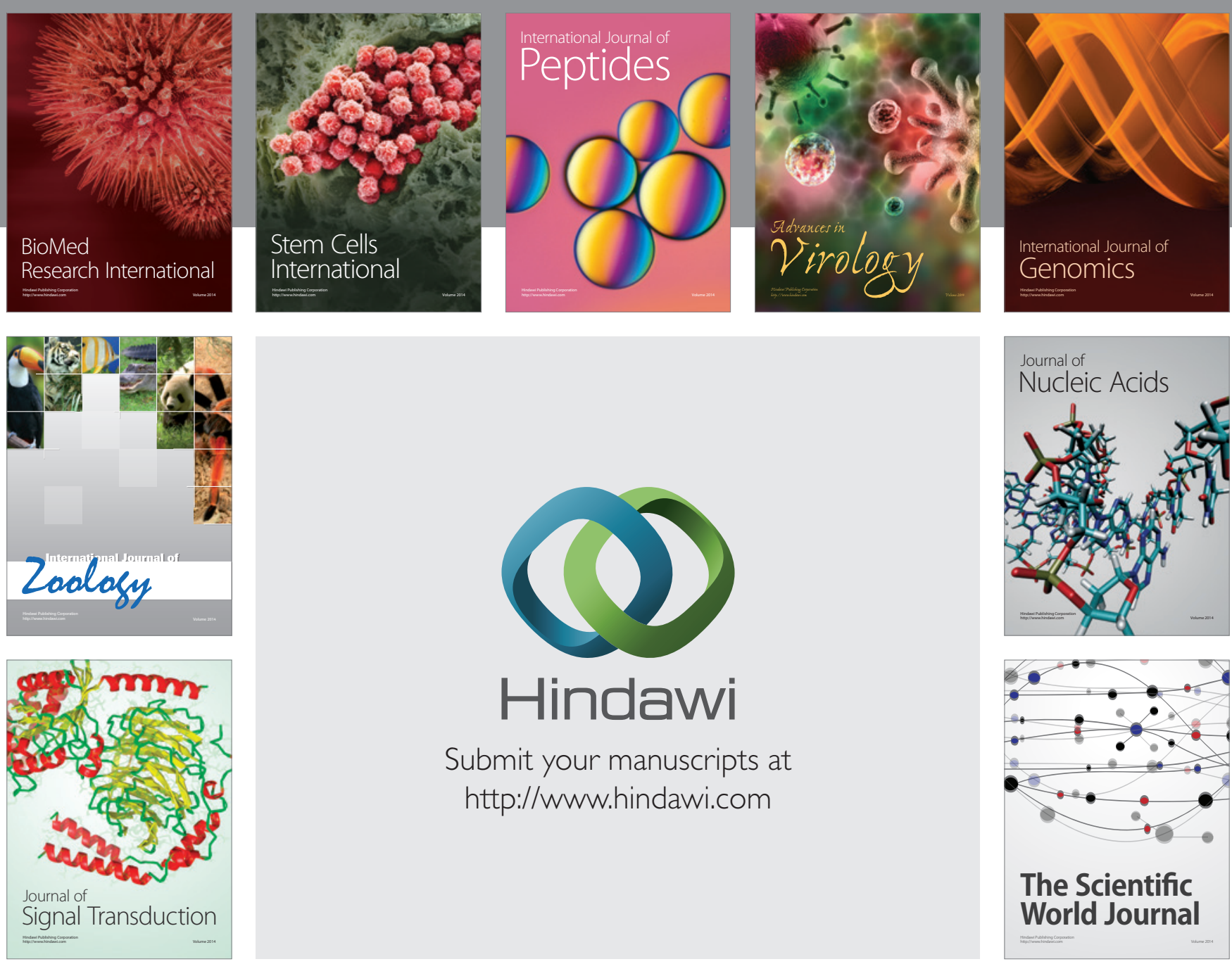

Submit your manuscripts at

http://www.hindawi.com
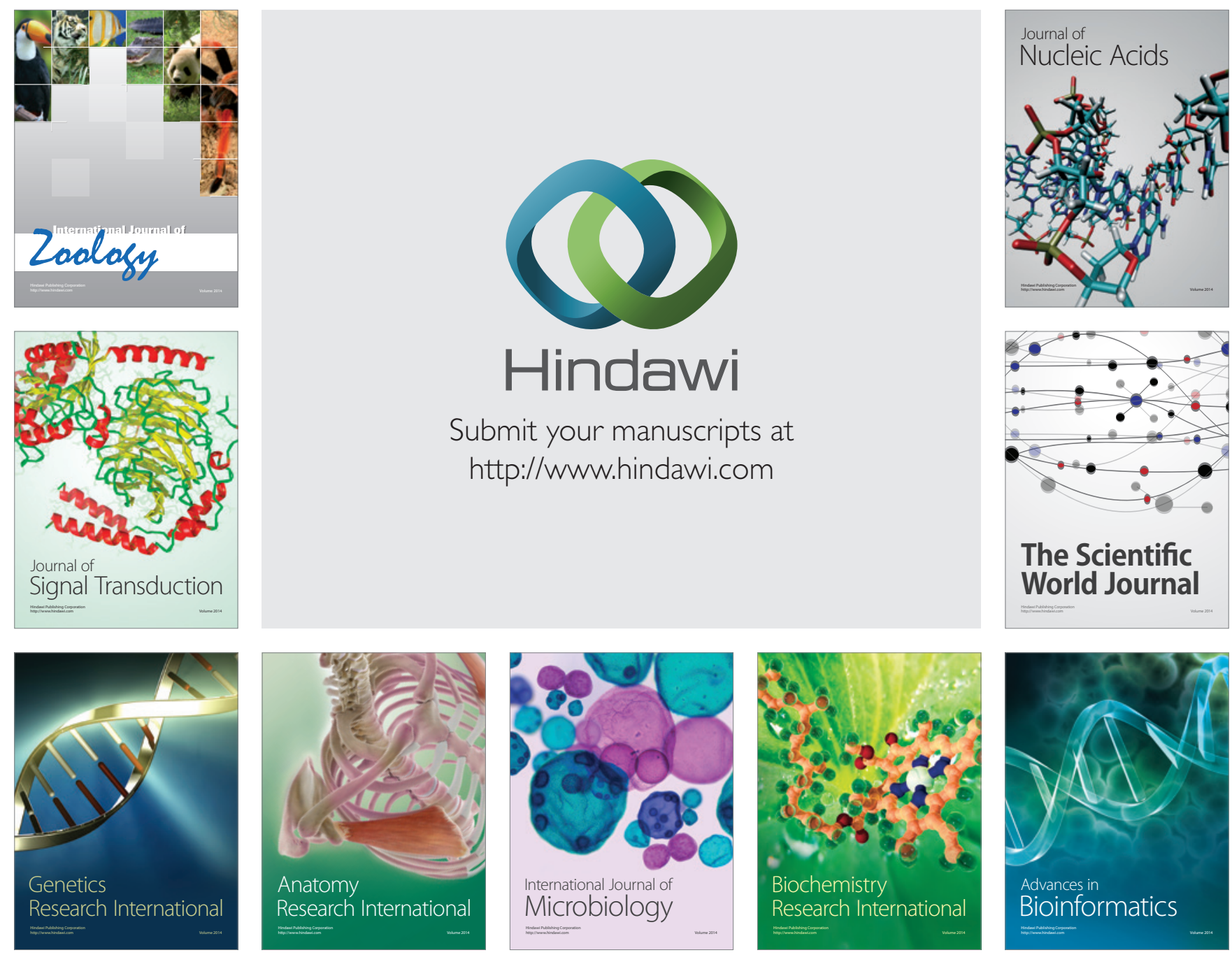

The Scientific World Journal
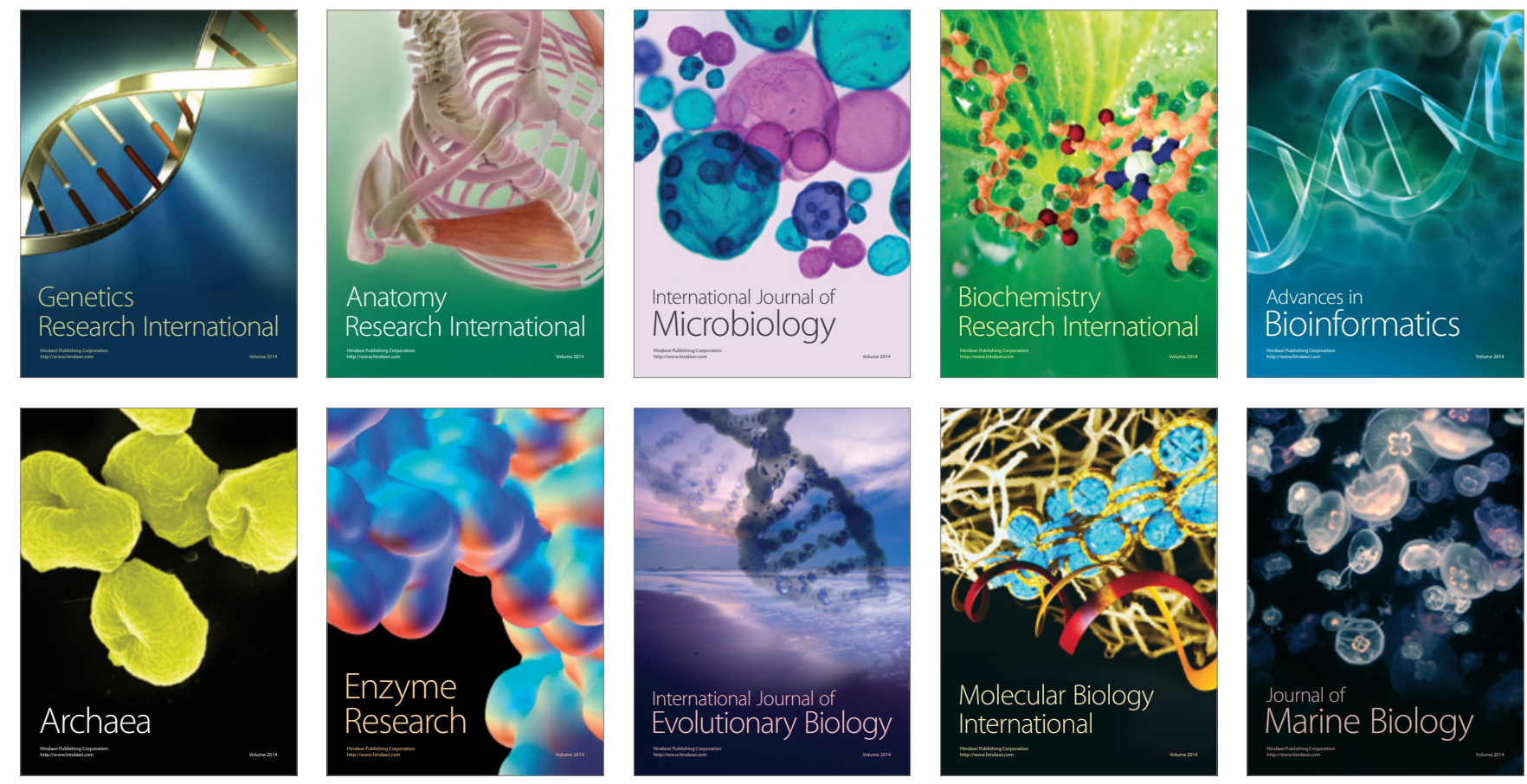